\section{Matrix, alternative}

T. Arndt

Bioscientia Institut für Medizinische Diagnostik $\mathrm{GmbH}$, Ingelheim, Deutschland

Synonym(e) Alternativmatrix

Englischer Begriff alternative matrix; complementary matrix

Definition Nicht näher definierter Begriff für Untersuchungsmaterial jenseits von Blut und seinen Produkten sowie Urin als häufigste Matrices des klinisch-chemischen Labors.

Beschreibung Die technologischen Entwicklungen der letzten Jahrzehnte erschlossen auch dem klinisch-chemischen und forensisch-toxikologischen Labor biologische Matrices, die vorher nur ungenügend oder unvollständig analytisch zugängig waren. Hierzu gehören in alphabetischer Reihenfolge Atemluft, Einzelhaare, Erbrochenes, Glaskörperflüssigkeit, Haare (Bart, Körper, Kopf, Scham), Fruchtwasser, Gewebe, Herzkammer- und Hohlvenenblut, Insekten (auf Leichen), Knochen, Liquor, Mageninhalt, Muttermilch, Mundflüssigkeit, Nagelmaterial (Hand, Fuß), Ohrenschmalz, Organe, Schweiß, Speichel, Sperma, Stuhl, Tränenflüssigkeit, Zähne.

Der Einsatz einiger Matrices wird sich weiter auf wenige Indikationen, ggf. Einzelfälle der forensischen Toxikologie, beschränken. Andere Matrices kommen vergleichsweise häufig und in weiter gefassten Indikationen zur Analyse, z. B. Liquor, Haare, Nagelmaterial, Speichel bzw. Mundflüssigkeit, Sperma.

Alternative Matrices bieten nicht nur eine Fülle an diagnostischen Informationen, sondern noch immer ein weites Forschungsfeld in Bezug auf Präanalytik, Analytik inkl. Qualitätssicherung und Interpretation. 